\title{
Impacto nas propriedades mecânicas do concreto curado sob baixas temperaturas
}

\author{
Impact on the mechanical properties \\ of concrete cured at low temperatures
}

Rafael Müller München ${ }^{1}$, Hinoel Zamis Ehrenbring ${ }^{1}$ Alessandra Wirth ${ }^{1}$, Daniel Reis Medeiros ${ }^{1}$, Bernardo Fonseca Tutikian ${ }^{1}$

\footnotetext{
${ }^{1}$ Universidade do Vale do Rio dos Sinos, UNISINOS, São Leopoldo, Rio Grande do Sul, Brasil. e-mail: rafaelmunchen87@gmail.com, hzamis@unisinos.br, alewirth@hotmail.com,drmedeiros@unisinos.br, bftutikian@unisinos.br
}

\begin{abstract}
RESUMO
O processo de cura das matrizes cimentícias é muito importante na determinação das suas propriedades no estado endurecido. Nessas circunstâncias, o concreto pode sofrer sérias avarias quando exposto às severas variações térmicas. As baixas temperaturas de cura em que as matrizes cimentícias são expostas podem influenciar nas propriedades no estado endurecido, principalmente nas idades iniciais, prejudicando os processos de desfôrma e escoramento. Locais de frio intenso devem seguir procedimentos específicos de dosagem e aplicação de uma matriz cimentícia. Sendo assim, essa pesquisa objetivou analisar a influência da temperatura de cura no comportamento mecânico e físico de concretos em diversas temperaturas de cura, tanto nas idades inicias como nas mais avançadas. Observou-se que a diminuição na temperatura de cura proporcionou aumento de resistência em até $26 \%$ na idade de 28 dias nos concretos analisados, apesar de que apresentaram desenvolvimento lento das propriedades mecânicas nos 7 dias iniciais. Concretos com maior consumo de cimento apresentaram melhor desempenho nas idades inicias quando submetidos às temperaturas menores que $3{ }^{\circ} \mathrm{C}$. Porém, percebeu-se que a cura em baixas temperaturas é capaz de proporcionar misturas mais íntegras em idades avançadas, visto que atingiram maior velocidade de propagação onda ultrassônica e resistência, pelo fato de ocorrer a hidratação residual do cimento.
\end{abstract}

Palavras-chave: Concreto, cura em baixa temperatura, propriedades mecânicas.

\begin{abstract}
The curing process of cementitious matrices is very important in determining their properties in the hardened state. In these circumstances, concrete can suffer serious damage when exposed to severe thermal variations. The low curing temperatures in which the cementitious matrices are exposed can influence their properties in the hardened state, especially in the early ages, impairing the processes of shaping and shoring. Intense cold places must follow specific dosing procedures and application of a cementitious matrix. Thus, this research aimed to analyze the influence of curing temperature on the mechanical and physical behavior of concretes at different curing temperatures, both at the early and later ages. It was observed that the decrease in curing temperature provided an increase of resistance up to $26 \%$ at the age of 28 days in the analyzed concretes, even though they presented slow development of the mechanical properties in the initial 7 days. Concretes with higher cement consumption showed better performance in early ages when submitted to temperatures lower than $3{ }^{\circ} \mathrm{C}$. However, it was observed that curing at low temperatures is able to provide more intact mixtures at advanced ages, since they achieved higher ultrasonic wave propagation velocity and resistance, due to the residual hydration of the cement.
\end{abstract}

Keywords: Cure of low temperature, mechanical properties.

\section{INTRODUÇÃO}

Estruturas de concreto são frequentemente expostas em ambientes e condições agressivas. Nessas situações, estão submetidas à inúmeras adversidades, passando pela ação de baixas e altas temperaturas, umidade 
relativa do ar, vento, entre outras [1,2]. As condições apresentadas variam com a localidade da estrutura e com a estação do ano, tendo como condições extremas o inverno e verão [3,4]. Todavia, no estado endurecido, o concreto é considerado um material resistente às intempéries e ameaças física [5,6]. As consequências mais severas pela variação da temperatura apresentam-se na etapa do lançamento do concreto e nos primeiros dias de idade, visto que está no estado fresco $[7,8]$. Logo, a concepção da composição de uma matriz cimentícia deve prever as variações que o material venha a sofrer, prospectando uma robustez adequada [9].

Sabe-se que as interferências sobre os concretos no estado fresco envolvem a retirada da água de hidratação da pasta cimentícia e suas consequências já são conhecidas, como: fissuração superficial e redução das propriedades mecânicas [10]. O congelamento de concretos é um processo gradativo, por depender da velocidade de transferência de calor, bem como da influência do tamanho dos poros e da quantidade de sais dissolvidos [11]. A água permanece na matriz e a atividade de suas moléculas é retardada, dificultando as reações de hidratação dos grãos de cimento anidros, o que promove ganhos lentos de resistência [12, 13]. Esse ganho pode ser considerado um problema para sistemas que objetivam construções rápidas, como a indústria de pré-fabricados. Dessa maneira, os principais problemas de concretagem em climas frios são operacionais [14].

Nas idades iniciais, a velocidade de hidratação dos grãos de cimento é influenciada pelo tipo de cimento, adições pozolânicas, quantidade de água, dimensão das partículas e, principalmente, pela temperatura no processo de cura [15]. Segundo Castro [16], temperaturas elevadas aceleram o processo de cura do concreto, possibilitando a melhoria das propriedades mecânicas em um curto período de tempo. Porém, Kraai [17] alerta que tal condição eleva a taxa de evaporação de água da pasta cimentícia e causa redução na efetividade de hidratação dos grãos. Já em baixas temperaturas, não há preocupação pela perda de umidade da pasta, mas sim pelo congelamento da água no interior da mistura, o que pode provocar atraso de pega, fissuração por expansão e vazios de concretagem [18-21]. As moléculas da água, quando submetidas às temperaturas próximas de $0^{\circ} \mathrm{C}$, tendem a se aglomerar, impossibilitando a movimentação livre dentro da pasta de cimento e perdendo o estado de fluidez, o que resulta na dificuldade de chegar aos grãos de cimento [22].

As propriedades da mistura curada sob baixas temperaturas foram estudadas, inicialmente, por Powers e Helmuth [23]. A partir desses, o comportamento das matrizes cimentícias nessas circunstâncias são entendidas por dois indicadores, a geração da pressão hidráulica e a difusão da água gel. A geração da pressão hidráulica é explicada pela água contida nos capilares do concreto que passa do estado líquido para o sólido e exerce uma pressão interna sobre as paredes do vazio, devido o aumento de volume. Dessa maneira, o movimento migratório da água é prejudicado até encontrar os grãos de cimento [14]. Já a difusão capilar é causada pela variação dos estados da água dentro dos poros, sendo que a solidificação inicia nos poros menores e favorece a migração da água-gel aos maiores vazios. Esse fenômeno demanda um maior nível de energia para movimentação da umidade no interior da pasta.

Dessa forma, o processo de ganho de resistência é mais prejudicado nas idades iniciais da estrutura. Esse comportamento pode restringir o uso do concreto em estruturas no inverno, principalmente pelo fato da desfôrma e da retirada do escoramento da estrutura serem postergadas [24, 25]. Após transcorrer um período do lançamento e haver aumento da temperatura, as moléculas de água começam a se movimentar pelo sistema e atingir os grãos de cimento anidro, o que ocasiona a hidratação residual e melhora o desempenho matriz nas idades avançadas [15, 26].

Chakraborty et al. [27] verificaram que o processo de pega do concreto, início e fim do endurecimento da pasta, é desacelerado em baixas temperaturas, pois a taxa de hidratação do $\mathrm{C}_{3} \mathrm{~S}$ é fortemente influenciada, diminuindo a sua produção e limitando a resistência mecânica nos primeiros dias. $\mathrm{O}$ uso de adições pozolânicas e materiais minerais suplementares em compósitos cimentícios pode trazer ganhos ao longo do tempo, pois estes são ativos em idades avançadas [28]. Os materiais pozolânicos necessitam ser ativados após a criação dos produtos de hidratação do cimento, assim, não são apropriados para ganhos de resistência nas idades inicias [29]. Analisando fisicamente e quimicamente o comportamento de uma matriz cimentícia em climas frios, observa-se que os materiais pozolânicos não são os mais indicados para utilização em concretos submetidos às baixas temperaturas para auxiliar no ganho de resistência inicial [5].

Em baixas temperaturas, tendo o concreto passado para o estágio viscoelástico, a expansão das moléculas de água atinge um aumento de $10 \%$ em relação ao seu volume original, proporcionando altas taxas de fissuração, devido à restrição no movimento [30, 31]. Essa condição é atingida em temperaturas próximas e abaixo de $0{ }^{\circ} \mathrm{C}$. Como fator ponderador, Powers [32] já indicava a incorporação de ar nesses compósitos em quantidades superiores a $7 \%$. Mesmo com todas as dificuldades físicas impostas pela temperatura, pesquisas evidenciam ganhos de resistências finais de concretos condicionados às temperaturas inferiores a $5^{\circ} \mathrm{C}[33$ 36]. 
Diante das limitações do concreto em baixas temperaturas, o ACI 306 [37] e EHE 08 [38] expõem as condições mínimas e orientações para concretagens em condições extremas de temperatura. De acordo com o ACI 306, não são recomendadas concretagens com a temperatura do ar abaixo de $4{ }^{\circ} \mathrm{C}$ ou quando a temperatura máxima diária não ultrapasse $10^{\circ} \mathrm{C}$ durante 12 horas por 3 dias consecutivos. Já o documento espanhol EHE 08 indica a temperatura de $5^{\circ} \mathrm{C}$ como condição crítica para a mistura em clima frio. Também, a ABNT NBR 14931 [39] frisa que concretagens em baixas temperaturas devem ser realizadas em temperatura ambiente de, no mínimo, $5^{\circ} \mathrm{C}$, caso contrário, o responsável técnico deve assumir a responsabilidade por falhas futuras.

Muitas vezes, nessas condições, a falta de cuidados pode causar danos no ciclo operacional das obras, bem como baixa resistência à compressão, baixo módulo de elasticidade, manifestações patológicas irreversíveis, devido aos problemas envolvendo a resistência inicial da matriz [15, 40, 41]. No entanto, procedimentos podem e devem ser adotados para mitigar os efeitos físicos no início e fim de pega do cimento [42]. Para Hawkes [43], o consumo de cimento na dosagem de concretos é uma das opções para que se mantenha as propriedades da mistura sob baixas temperaturas.

Para tanto, elaborou-se essa pesquisa com o intuito de verificar as propriedades mecânicas e comportamento físico do concreto, utilizando diferentes composições, submetidas em condições de cura com temperatura de $-3,0,3,8$ e $22^{\circ} \mathrm{C}$, simulando diferentes condições climáticas e idades distintas. $\mathrm{O}$ estudo se deu com três diferentes classes de concreto, para observar o comportamento de misturas ricas, intermediárias e pobres, correlacionando a resistência à compressão e tração com a velocidade de propagação de ondas ultrassônicas.

\section{MATERIAIS E MÉTODOS}

O programa experimental da pesquisa contou com ensaios e métodos normalizados, sendo eles: ensaio de resistência à compressão axial, resistência à tração por compressão diametral e determinação da velocidade de propagação de onda ultrassônica. Examinaram-se três composições de concreto, sendo a rica $(1: 3,5)$, a intermediária $(1: 5,0)$ e outra pobre (1: 6,5). Dessa forma, o programa experimental foi elaborado para determinar as propriedades mecânicas dos compósitos quando submetidos às variações na temperatura de cura. No total foram moldados 105 corpos de prova cilíndricos de 10 x $20 \mathrm{~cm}$.

A resistência à compressão axial dos concretos e a velocidade de propagação de onda ultrassônica foram determinadas no $1^{\circ}, 7^{\circ}$ e $28^{\circ}$ dia de idade. Já para a resistência à tração, a idade adotada foi de 28 dias. A investigação para cada traço contou com 35 amostras, sendo 7 analisadas para cada temperatura especificada. As condições de cura da pesquisa foram referentes às baixas temperaturas, expondo as amostras de concreto a $-3,0,3,8{ }^{\circ} \mathrm{C}$ e a temperatura referência de $22{ }^{\circ} \mathrm{C}$. A escolha das temperaturas de cura teve como base as condições climáticas encontradas na região sul do Brasil, onde é possível atingir valores próximos ou abaixo de $0{ }^{\circ} \mathrm{C}$. As temperaturas de $-3,0$ e $3{ }^{\circ} \mathrm{C}$ foram escolhidas para ter pontos abaixo da temperatura inferior limite de normas nacionais e internacionais (ABNT 14931, ACI 306, EHE 08) recomendadas para concretagens, de $5^{\circ} \mathrm{C}$. Ainda, se definiu um ponto ligeiramente acima do limite de $5^{\circ} \mathrm{C}$, o de $8{ }^{\circ} \mathrm{C}$, e a referência, de $22^{\circ} \mathrm{C}$. Os concretos foram curados em câmara fria, nas respectivas temperaturas, durante os primeiros 7 dias de cura. Já no restante do período, os 21 dias, mantiveram-se em condições conforme ABNT NBR 5738 [44]. Os exemplares com a temperatura de $22{ }^{\circ} \mathrm{C}$ foram condicionados em tempo integral às condições de norma. $\mathrm{O}$ teor de umidade mantido na câmara fria e na convencional foi de 95 $\pm 3 \%$.

\subsection{Materiais utilizados}

A seguir foram elencados os materiais utilizados nas composições estudadas. Os insumos que compuseram as misturas são encontrados no Rio Grande do Sul, Brasil.

\subsubsection{Cimento}

Como aglomerante hidráulico, utilizou-se o cimento Portland CP V - ARI (alta resistência inicial), e sua composição química está expressa na Tabela 1 . A massa específica é de $3,08 \mathrm{~g} / \mathrm{cm}^{3}$, determinada pela ABNT NBR NM 23 [45]. 
Tabela 1: Análise química do cimento

\begin{tabular}{c|c}
\hline COMPONENTE & VALOR (\%) \\
\hline $\mathrm{SiO}_{2}$ & 23,18 \\
\hline $\mathrm{Al}_{2} \mathrm{O}_{3}$ & 6,42 \\
\hline $\mathrm{Fe}_{2} \mathrm{O}_{3}$ & 3,36 \\
\hline $\mathrm{CaO}$ & 51,6 \\
\hline $\mathrm{MgO}$ & 5,96 \\
\hline $\mathrm{CO}_{2}$ & 2,45 \\
\hline
\end{tabular}

\subsubsection{Agregado miúdo}

O agregado miúdo escolhido foi areia natural de origem quartzosa, extraída de rio. A massa unitária do agregado é de $1,60 \mathrm{~g} / \mathrm{cm}^{3}$, sendo determinada pela ABNT NBR NM 45 [46]. A massa específica é de 2,55 $\mathrm{g} / \mathrm{cm}^{3}$ e foi determinada seguindo os parâmetros da ABNT NBR 52 [47]. A composição granulométrica dos agregados miúdos é apresentada na Tabela 2 e Figura 1, seguindo as determinações da ABNT NBR NM 23 [45] e ABNT NBT 7211 [48].

Tabela 2: Composição granulométrica da areia

\begin{tabular}{l|c|c}
\hline $\begin{array}{c}\text { ABERTURA DE } \\
\text { PENEIRA }\end{array}$ & \% RETIDO & \% ACUMULADO \\
\hline $9,5 \mathrm{~mm}$ & 0,0 & 0,0 \\
\hline $6,3 \mathrm{~mm}$ & 1,0 & 1,0 \\
\hline $4,8 \mathrm{~mm}$ & 1,0 & 2,0 \\
\hline $2,4 \mathrm{~mm}$ & 5,0 & 6,0 \\
\hline $1,2 \mathrm{~mm}$ & 12,0 & 19,0 \\
\hline $0,6 \mathrm{~mm}$ & 22,0 & 40,0 \\
\hline $0,3 \mathrm{~mm}$ & 44,0 & 84,0 \\
\hline $0,15 \mathrm{~mm}$ & 15,0 & 100,0 \\
\hline$<0,15 \mathrm{~mm}$ & 0,0 & 100,0 \\
\hline$\Sigma$ & 100,0 & 100,0 \\
\hline Módulo de finura & & 2,51 \\
\hline Dimensão máxima (mm) & 4,80 \\
\hline
\end{tabular}

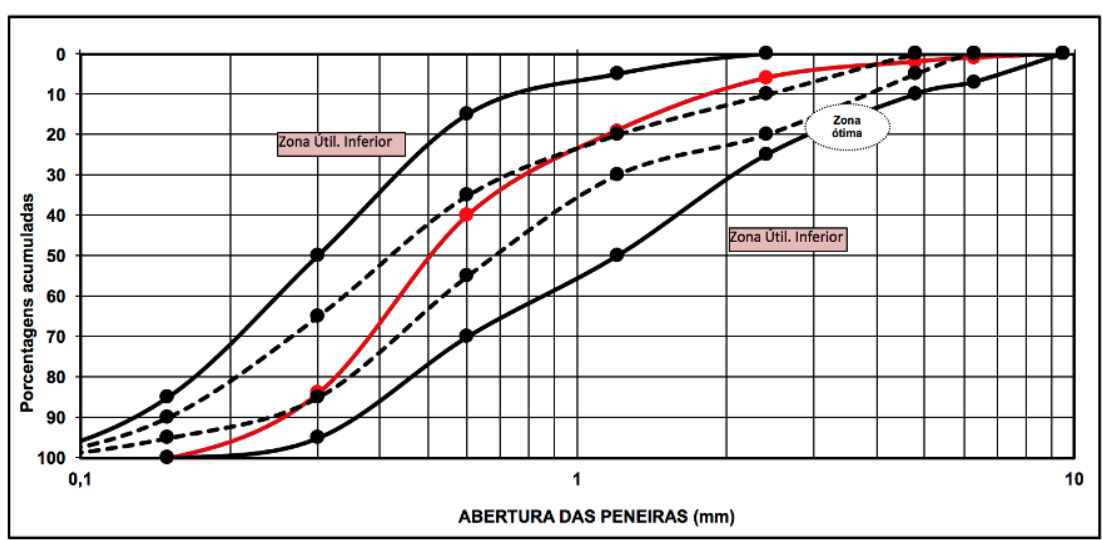

Figura 1: Curva granulométrica do agregado miúdo

\subsubsection{Agregado graúdo}

O agregado graúdo utilizado é de origem basáltica e extraído na cidade de Montenegro, Rio Grande do Sul, Brasil. A massa específica do material foi determinada por meio da ABNT NBR NM 53 [49], correspondendo a $2,58 \mathrm{~g} / \mathrm{cm}^{3}$. Já a massa unitária é equivalente a $1,41 \mathrm{~g} / \mathrm{cm}^{3}$ e foi determinada pelo método 
proposto na ABNT NBR NM 45 [46]. A composição granulométrica dos agregados miúdos é apresentada na Tabela 3 e Figura 2, seguindo as determinações da ABNT NBR NM 23 [45] e ABNT NBT 7211 [48].

Tabela 3: Composição granulométrica do agregado graúdo

\begin{tabular}{|c|c|c|}
\hline $\begin{array}{c}\text { ABERTURA DE } \\
\text { PENEIRA }\end{array}$ & \% RETIDO & \% ACUMULADO \\
\hline $25,0 \mathrm{~mm}$ & 0,0 & 0,0 \\
\hline $19,0 \mathrm{~mm}$ & 0,0 & 0,0 \\
\hline $12,5 \mathrm{~mm}$ & 0,0 & 0,0 \\
\hline $9,5 \mathrm{~mm}$ & 0,6 & 0,6 \\
\hline $6,3 \mathrm{~mm}$ & 29,1 & 29,7 \\
\hline $4,8 \mathrm{~mm}$ & 32,7 & 62,4 \\
\hline$<4,8 \mathrm{~mm}$ & 37,6 & 100,0 \\
\hline$\Sigma$ & 100,0 & 100,0 \\
\hline \multicolumn{2}{|l|}{ Módulo de finura } & 6,63 \\
\hline \multicolumn{2}{|c|}{ Dimensão máxima (mm) } & 9,50 \\
\hline
\end{tabular}

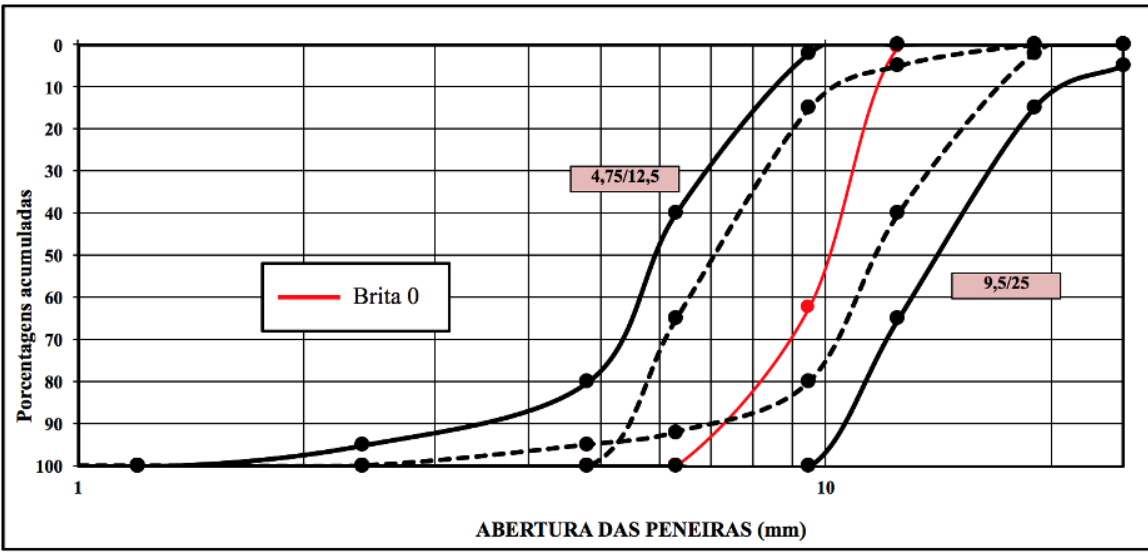

Figura 2: Curva granulométrica do agregado graúdo utilizado

\subsection{Método de dosagem}

A dosagem dos concretos foi realizada com base no método Tutikian e Helene [50], conhecido como método Ibracon. Determinou-se 3 traços para obter a curva de dosagem da matriz, sendo o traço unitário do pobre (1: $6,5)$, o do intermediário $(1: 5,0)$ e do rico $(1: 3,5)$. O teor de argamassa foi fixado em $52 \%$, com relação água/cimento variável. A classe abatimento estipulada foi a S100 da ABNT NBR 7212 [51]. A Tabela 4 apresenta a descriminação das misturas.

Tabela 4: Dosagem dos traços determinados

\begin{tabular}{c|c|c|c|c|c|c}
\hline \multicolumn{2}{c|}{ TRAÇOS UNITÁRIOS } & $\begin{array}{c}\text { CIMENTO } \\
\left(\mathbf{k g} / \mathbf{m}^{\mathbf{3}}\right)\end{array}$ & $\begin{array}{c}\text { AREIA } \\
\left(\mathbf{k g} / \mathbf{m}^{\mathbf{3}}\right)\end{array}$ & $\begin{array}{c}\text { BRITA } \\
\left(\mathbf{k g} / \mathbf{m}^{\mathbf{3}}\right)\end{array}$ & $\begin{array}{c}\text { RELAÇÃo } \\
\mathbf{a} / \mathbf{c}\end{array}$ & $\begin{array}{c}\text { CLASSE DE } \\
\text { ABATIMENTO } \\
(\mathbf{m m})\end{array}$ \\
\hline Rico (1:3,5) & $1: 1,34: 2,16$ & 488,0 & 654,0 & 1054,0 & 0,42 & \multirow{2}{*}{$\mathrm{S} 100$} \\
\cline { 1 - 5 } Intermediário (1:5,0) & $1: 2,12: 2,88$ & 367,0 & 779,0 & 1057,0 & 0,53 & \\
\cline { 1 - 5 } Pobre (1: 6,5 & $1: 2,90: 3,60$ & 295,0 & 855,0 & 1062,0 & 0,64 & \\
\hline
\end{tabular}

\subsection{Ensaios realizados}

Os ensaios realizados para a determinação das propriedades mecânicas dos concretos foram a resistência à compressão, resistência à tração por compressão diametral e a determinação da velocidade de propagação de ondas ultrassônicas. Para a execução desses métodos, utilizou-se amostras cilíndricas moldadas, conforme recomenda a ABNT NBR 5738 [44]. 


\subsubsection{Ensaios de resistência à compressão axial}

O ensaio para determinação da resistência à compressão axial seguiu a ABNT NBR 5739 [52]. A velocidade do ensaio foi de $0,30 \mathrm{MPa} / \mathrm{s}$ até atingir a ruptura da amostra, conforme determina a norma. A prensa utilizada tem capacidade para efetuar um carregamento de até $2000 \mathrm{kN}$.

Ainda, diante do comportamento das misturas em relação a sua condição de cura, correlacionou-se a resistência à compressão com o seu grau de maturidade (GM), conforme Equação (1) de Nurse-Saul ACI 325 [53].

$$
G M=\Sigma\left(T_{a}-T_{0}\right) * t
$$

Onde:

$\mathrm{GM}=$ grau de maturidade $\left({ }^{\circ} \mathrm{C} * \mathrm{dia}\right)$

$\mathrm{T}_{\mathrm{a}}=$ temperatura de cura $\left({ }^{\circ} \mathrm{C}\right)$;

$\mathrm{T}_{0}=$ temperatura de referência $\left({ }^{\circ} \mathrm{C}\right)$;

$\mathrm{t}=$ tempo em que a amostra foi submetida a determinada temperatura.

O método de análise adotando o grau de maturidade visa comparar a temperatura de exposição e o tempo em que a amostra permaneceu submetida sob tal circunstância. Dessa maneira, quanto menor a temperatura, menor será o grau de maturidade, ou seja, maior a dificuldade de efetivar a hidratação dos aglomerantes hidráulicos. Concretos que possuem o menor grau de maturidade tendem a obter menores resistências. Dessa maneira, afim de equalizar os parâmetros de avaliação, determinou-se que os fatores comparativos entre as misturas seriam pelo método do GM.

A temperatura de referência é a de $-10^{\circ} \mathrm{C}$, então aplicou-se tal valor para embasar a equação, resultando na Equação 2. A elaboração das curvas foi feita com base no tempo de condicionamento e a temperatura de exposição das amostras, definindo o grau de maturidade da mistura.

$$
G M=\Sigma\left(T+10^{\circ} \mathrm{C}\right) * t
$$

Onde:

$\mathrm{GM}=$ grau de maturidade $\left({ }^{\circ} \mathrm{C} * \mathrm{dia}\right)$;

$\mathrm{T}=$ temperatura de cura $\left({ }^{\circ} \mathrm{C}\right)$;

$\mathrm{t}=$ tempo em que a amostra foi submetida a determinada temperatura.

\subsubsection{Resistência à tração por compressão diametral}

Para a realização dos ensaios foram seguidas as recomendações da ABNT NBR 7222 [54], utilizando-se corpos de prova cilíndricos com dimensões iguais a 10 x $20 \mathrm{~cm}$. Os exemplares foram colocados horizontalmente entre os pratos e o pistão de carregamento da prensa, sendo aplicada uma tensão constante de $0,05 \mathrm{MPa} / \mathrm{s}$, até sua ruptura por tração indireta. A prensa utilizada foi a mesma do ensaio de resistência à compressão axial.

\subsubsection{Determinações da velocidade de onda ultrassônica}

Para a análise da velocidade de onda ultrassônica nas amostras estudadas, o método de ensaio baseou-se na IS 13311 [55] e ABNT NBR 8802 [56]. O ensaio foi realizado com equipamento de ultrassom fabricado pela Proceq Ltda., o qual possui duas sondas, uma emissora e outra receptora. Nesse caso, os exemplares foram analisados pelo método direto, onde as sondas foram posicionadas nas faces opostas dos mesmos e distantes $20 \mathrm{~cm}$ entre si. Os parâmetros qualitativos referentes à velocidade da onda basearam-se na Tabela 5, desenvolvida por Whitehurst [57].

Tabela 5: Qualidade do concreto em relação à velocidade de propagação de ondas ultrassônicas

\begin{tabular}{c|c}
\hline $\begin{array}{c}\text { VELOCIDADE DE PROPAGAÇÃO DE } \\
\text { ONDAS ULTRASSONICAS }(\mathbf{m} / \mathbf{s})\end{array}$ & $\begin{array}{c}\text { QUALIDADE DO } \\
\text { CONCRETO }\end{array}$ \\
\hline Acima de 4500 & Excelente \\
\hline $3500-4500$ & Ótimo \\
\hline $3000-3500$ & Bom \\
\hline $2000-3000$ & Regular \\
\hline Inferior -2000 & Ruim \\
\hline
\end{tabular}




\section{RESULTADOS}

\subsection{Resistência à compressão axial}

A Tabela 6 ilustra os valores obtidos nos ensaios de resistência à compressão axial potencial para todas as misturas.

Tabela 6: Resistência à compressão axial potencial das composições

\begin{tabular}{c|c|c|c|c|c|c|c|c|c}
\hline \multirow{2}{*}{$\begin{array}{c}\text { TEMPERATURA } \\
\text { DE CURA } \\
\left({ }^{\circ} \mathbf{C}\right)\end{array}$} & \multicolumn{6}{|c|}{ RESISTÊNCIA À COMPRESSÃO AXIAL POTENCIAL (MPa) } \\
\cline { 2 - 10 } & $\begin{array}{c}\mathbf{1} \\
\mathbf{d i a}\end{array}$ & $\begin{array}{c}\mathbf{7} \\
\text { dias }\end{array}$ & $\begin{array}{c}\mathbf{2 8} \\
\text { dias }\end{array}$ & $\begin{array}{c}\mathbf{1} \\
\mathbf{d i a}\end{array}$ & $\begin{array}{c}\mathbf{7} \\
\text { dias }\end{array}$ & $\begin{array}{c}\mathbf{2 8} \\
\text { dias }\end{array}$ & $\begin{array}{c}\mathbf{1} \\
\text { dia }\end{array}$ & $\begin{array}{c}\mathbf{7} \\
\text { dias }\end{array}$ & $\begin{array}{c}\mathbf{2 8} \\
\text { dias }\end{array}$ \\
\hline 22,0 & 20,6 & 31,4 & 37,6 & 17,1 & 24,3 & 33,7 & 13,6 & 23,7 & 26,8 \\
\hline 8,0 & 19,2 & 30,5 & 42,5 & 11,1 & 22,3 & 35,3 & 4,6 & 18,8 & 24,3 \\
\hline 3,0 & 9,6 & 29,9 & 38,2 & 3,5 & 19,0 & 36,1 & 3,3 & 17,5 & 23,8 \\
\hline 0,0 & 6,9 & 29,8 & 42,7 & 1,3 & 18,5 & 38,0 & 0,0 & 16,7 & 25,8 \\
\hline$-3,0$ & 7,9 & 28,7 & 47,2 & 1,5 & 21,6 & 40,0 & 0,4 & 16,0 & 26,5 \\
\hline
\end{tabular}

Conforme expressa a Tabela 6, os resultados de resistência à compressão atingidos pelo traço rico foram os mais elevados da pesquisa, independentemente da temperatura de cura. Também foi possível identificar que as amostras ensaiadas no $1^{\circ}$ e $7^{\circ}$ dia de idade tiveram a resistência inferior aos 28 dias. Os piores resultados nas idades inicias foram, com a temperatura de -3 e $0{ }^{\circ} \mathrm{C}$, como já era esperado, sendo também verificado por Liu et al. [33]. As amostras curadas com temperatura de $22{ }^{\circ} \mathrm{C}$, em todos os traços, atingiram as maiores resistências nas idades inicias $\left(1^{\circ}\right.$ e $7^{\circ}$ dia), devido às condições de cura distintas nos primeiros sete dias. À medida que a temperatura de cura foi superior a $3{ }^{\circ} \mathrm{C}$, as melhoras na resistência à compressão dos traços foram evidentes. O desenvolvimento de uma condição favorável não foi atingido em baixas temperaturas, pois as reações de hidratação são exotérmicas. Complementando, Chakraborty [27] afirma que a formação de $\mathrm{C}_{3} \mathrm{~S}$, produto de hidratação do cimento, é retardada, causando impacto na resistência inicial da matriz.

Nota-se que a temperatura de cura influencia na resistência à compressão das matrizes cimentícias, sendo o traço pobre o mais prejudicado, atingindo resistência nula no primeiro dia de ensaio nas temperaturas de 0 e $-3{ }^{\circ} \mathrm{C}$. Já o traço intermediário, para as mesmas condições, apresentou pequena melhora, conferindo resistências de 1,3 e 1,5 MPa. Segundo Lima e Libório [14] e Soriano et al. [15], as matrizes cimentícias possuem dificuldade no desenvolvimento das resistências inicias, devido à redução na velocidade de hidratação do cimento pela pressão hidráulica e difusão da água.

O grau de maturidade de cada concreto está apresentado na Tabela 7 e as curvas obtidas entre a relação de resistência à compressão e GM são apresentadas na Figura 3.

Tabela 7: Grau de maturidade (GM) referente as temperaturas analisadas

\begin{tabular}{c|c|c}
\hline $\begin{array}{c}\text { TEMPERATURA } \\
\left({ }^{\circ} \mathbf{C}\right)\end{array}$ & $\begin{array}{c}\text { IDADE } \\
(\mathbf{d i a s})\end{array}$ & $\begin{array}{c}\text { GM } \\
\left({ }^{\circ} \mathbf{C}^{*} \mathbf{d i a}\right)\end{array}$ \\
\hline \multirow{4}{*}{-3} & 1 & 7 \\
\cline { 2 - 3 } & 7 & 49 \\
\cline { 2 - 3 } & 28 & 721 \\
\hline \multirow{4}{*}{0} & 1 & 10 \\
\cline { 2 - 3 } & 7 & 70 \\
\cline { 2 - 3 } & 28 & 742 \\
\hline \multirow{4}{*}{3} & 1 & 13 \\
\cline { 2 - 3 } & 7 & 91 \\
\cline { 2 - 3 } & 28 & 763 \\
\hline \multirow{4}{*}{8} & 1 & 18 \\
\cline { 2 - 3 } & 7 & 126 \\
\cline { 2 - 3 } & 28 & 798 \\
\hline \multirow{4}{*}{22} & 1 & 32 \\
\cline { 2 - 3 } & 7 & 224 \\
\cline { 2 - 3 } & 28 & 896 \\
\hline
\end{tabular}




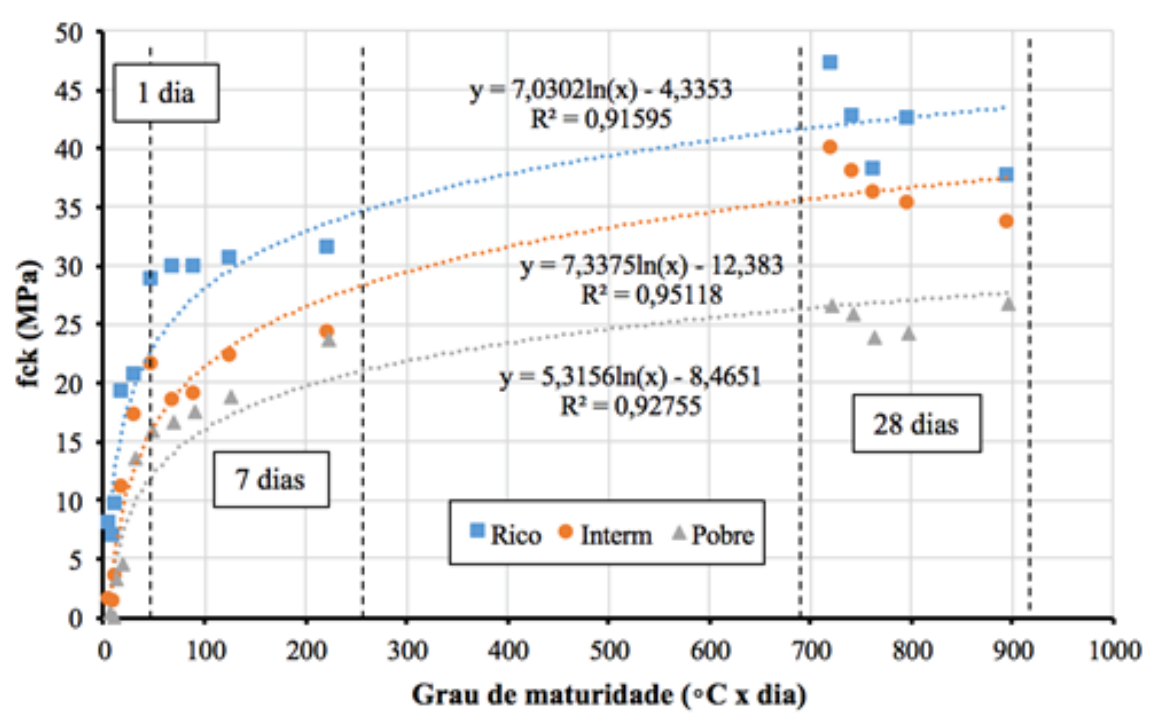

Figura 3: Ganhos de resistência à compressão em relação ao grau de maturidade

Conforme apresenta a Figura 3, contata-se a correlação elevada das curvas formadas por meio do grau de maturidade e resistência à compressão das misturas. Analisando a resistência atingida nos diferentes compósitos nas primeiras 24 horas, verifica-se que o GM é próximo a $50{ }^{\circ} \mathrm{C} *$ dia, tendo as maiores resistências concentradas nas temperaturas mais elevadas, ou seja, 8 e $22{ }^{\circ} \mathrm{C}$. As diferenças são evidentes, onde algumas amostras atingiram fc de $20 \mathrm{MPa}$ e outras apenas $0,4 \mathrm{MPa}$.

Porém, com o aumento do grau de maturidade, as amostras apresentaram evolução na resistência, independente das condições de cura. Nota-se que para o traço rico, mesmo com o GM das amostras condicionadas em baixas temperaturas, entre 50 e $120^{\circ} \mathrm{C} *$ dia, o fc manteve-se próximo a $30 \mathrm{MPa}$. Ou seja, nessa mistura, a diferença na condição de cura, na idade de 7 dias, não apresentou variação considerável entre as misturas mantidas abaixo de $8{ }^{\circ} \mathrm{C}$ e a $22{ }^{\circ} \mathrm{C}\left(\mathrm{GM}=224{ }^{\circ} \mathrm{C} *\right.$ dia $)$. Já no traço intermediário e pobre, à medida que a o GM diminuiu, a resistência também foi prejudicada. $\mathrm{O}$ comportamento de traços com maior consumo de cimento também se mostrou superior aos demais na pesquisa realizada por Yan et al. [58], pelo fato dessa matriz ter uma robustez maior e proporcionar uma maior taxa de hidratação devido o teor de cimento.

Comparando as amostras aos 28 dias, o comportamento é invertido. As misturas com maior grau de maturidade $\left(896^{\circ} \mathrm{C} *\right.$ dia) atingiram valores, na maioria dos casos, inferiores às amostras com GM entre $700 \mathrm{e}$ $800{ }^{\circ} \mathrm{C} *$ dia. Observa-se que, tanto no traço rico quanto no intermediário, à medida que se diminuiu o grau de maturidade, aumentou-se a resistência à compressão da mistura. No traço rico, a diferença entre a cura com temperatura de 22 e $-3{ }^{\circ} \mathrm{C}$ foi de, até, $26 \%$. Já no concreto com traço pobre, os valores mantiveram-se semelhantes, próximos de $25 \mathrm{MPa}$, mesmo com diferenças no GM. Portanto, nessas circunstâncias, é possível afirmar que as baixas temperaturas influenciam positivamente na resistência da matriz em idades avançadas, tendo proximidade ao comportamento das matrizes investigadas por $\mathrm{Xu}$ et al. [34].

Os valores para cada traço, após os 7 dias de idade, chegaram a obter um crescimento de $65 \%$ nas menores temperaturas. Já com $22{ }^{\circ} \mathrm{C}$, o ganho de resistência das misturas foi de $20 \%$. Após submetidos a um ambiente severo por 7 dias, as amostras foram realocadas a um ambiente com temperatura de $22{ }^{\circ} \mathrm{C}$, melhorando o processo de hidratação do cimento. Os elevados ganhos de resistência aos 28 dias das misturas condicionadas em baixas temperaturas são explicados pela redistribuição da umidade interna na mistura. Com o aumento da temperatura, a água interna passa pelo processo físico de fusão, aumentando a movimentação do fluido e atingindo os grãos de cimento ainda anidros [9]. Dessa forma, o processo de hidratação do aglomerante foi postergado pelo fenômeno da difusão água gel, sendo mais efetivo com a mudança das condições. Ainda se evidenciou que, em baixas temperaturas, a umidade interna do compósito não foi diminuída, conservando a mesma quantidade de água utilizada no processo de mistura, a qual se encontrou sob pressão hidráulica, conforme afirmaram Powers e Helmuth [23].

Percebe-se que, em todos os traços, o ganho de resistência foi elevado no final dos 28 dias, já que o processo de cura foi menos intenso nas baixas temperaturas, havendo uma maior dificuldade das partículas do cimento para absorção das moléculas de água em -3 e $0{ }^{\circ} \mathrm{C}$. A Tabela 8 apresenta os ganhos de resistência de cada traço em relação ao período de análise. 
Tabela 8: Equação de ganho de resistência

\begin{tabular}{|c|c|c|c|}
\hline TRAÇOS & $\begin{array}{l}\text { TEMPERATURA } \\
\text { DE CURA }\left({ }^{\circ} \mathbf{C}\right)\end{array}$ & $\begin{array}{c}\text { EQUAÇÃO DO } \\
\text { GANHO DE RESISTÊNCIA }\end{array}$ & $\mathbf{R}^{2}$ \\
\hline \multirow{5}{*}{$\begin{array}{l}\text { RICO } \\
(1: 3,5)\end{array}$} & 22,0 & $y=8,5 x+12,867$ & 0,97 \\
\hline & 8,0 & $y=11,65 x+7,4333$ & 0,99 \\
\hline & 3,0 & $y=14,3 x-2,7$ & 0,94 \\
\hline & 0,0 & $y=17,9 x-9,3333$ & 0,97 \\
\hline & $-3,0$ & $y=19,65 x-11,367$ & 0,99 \\
\hline \multirow{5}{*}{$\begin{array}{l}\text { INTERM. } \\
(1: 5,0)\end{array}$} & 22,0 & $y=8,3 x+8,4333$ & 0,99 \\
\hline & 8,0 & $y=5,6 x+7,3667$ & 0,99 \\
\hline & 3,0 & $y=16,3 x-13,067$ & 0,99 \\
\hline & 0,0 & $y=18,35 x-17,433$ & 0,99 \\
\hline & $-3,0$ & $y=19,25 x-17,467$ & 0,99 \\
\hline \multirow{5}{*}{$\begin{array}{l}\text { POBRE } \\
(1: 6,5)\end{array}$} & 22,0 & $y=6,575 x+8,2$ & 0,91 \\
\hline & 8,0 & $y=9,85 x-3,8$ & 0,94 \\
\hline & 3,0 & $y=10,25 x-5,6333$ & 0,95 \\
\hline & 0,0 & $y=12,9 x-11,633$ & 0,97 \\
\hline & $-3,0$ & $y=13,05 x-11,8$ & 0,98 \\
\hline
\end{tabular}

Conforme expressa a Tabela 8, os ganhos de resistência à compressão para os 3 traços ilustrados, em condições de $-3,0$ e $3{ }^{\circ} \mathrm{C}$, foram semelhantes entre si e superiores aos expostos para as temperaturas de $8{ }^{\circ} \mathrm{C}$ e $22{ }^{\circ} \mathrm{C}$. No traço rico $(1: 3,5)$, na temperatura de $-3{ }^{\circ} \mathrm{C}$, o ganho de resistência foi cerca de $130 \%$ maior quando comparado ao mesmo traço curado com $22{ }^{\circ} \mathrm{C}$. À medida que a temperatura de cura se elevou, os ganhos diminuíram. No traço intermediário $(1: 5,0)$, a $-3{ }^{\circ} \mathrm{C}$, também foi identificado resultados elevados, sendo 2 vezes maiores em relação aos $22^{\circ} \mathrm{C}$. No traço pobre $(1: 6,5)$, os ganhos de resistência foram menores em relação aos demais, porém, nas baixas temperaturas $\left(-3,0\right.$ e $\left.3{ }^{\circ} \mathrm{C}\right)$, o traço apresentou ganho de $100 \% \mathrm{em}$ função da condição de cura ideal. Com isso, verificou-se que à medida que as temperaturas de cura foram reduzidas, os ganhos de resistência elevaram-se, podendo ser duplicadas e ultrapassar as resistências potenciais aos 28 dias dos traços curados em temperaturas de $22{ }^{\circ} \mathrm{C}$, como também constatado por Xu et al. [34].

\subsection{Resistência à tração por compressão diametral}

Os resultados obtidos no ensaio de resistência à tração por compressão diametral estão apontados na Tabela 9. Os testes foram realizados aos 28 dias de idade.

Tabela 9: Resultados de resistência à tração por compressão diametral potencial aos 28 dias

\begin{tabular}{c|c|c|c}
\hline \multirow{2}{*}{$\begin{array}{c}\text { TEMPERATURA } \\
\text { DE CURA }\left({ }^{\circ} \mathbf{C}\right)\end{array}$} & \multicolumn{3}{|c}{$\begin{array}{c}\text { RESISTÊNCIA A TRAÇÃO POR COMPRESSÃO } \\
\text { DIAMETRAL POTENCIAL (MPa) }\end{array}$} \\
\cline { 2 - 4 } & RICO & INTERMEDIÁRIO & POBRE \\
\hline 22,0 & 3,3 & 3,2 & 2,8 \\
\hline 8,0 & 3,7 & 3,5 & 2,8 \\
\hline 3,0 & 3,8 & 3,6 & 2,8 \\
\hline 0,0 & 3,5 & 2,8 & 2,6 \\
\hline$-3,0$ & 4,4 & 4,1 & 3,0 \\
\hline
\end{tabular}

Observa-se, na Tabela 9, que as amostras curadas em temperatura de $-3{ }^{\circ} \mathrm{C}$ foram as que atingiram os maiores resultados. Quando se comparam os valores entre as condições de 22 e $-3{ }^{\circ} \mathrm{C}$, os ganhos de resistência para a baixa temperatura atingiram $33 \%$, para os traços rico e intermediário. 


\subsection{Velocidade de ondas ultrassônicas}

Os resultados obtidos nos ensaios de velocidade de ondas ultrassônicas em cada traço estão apresentados na Tabela 10. Essa propriedade mede a homogeneidade da mistura e a quantidade de vazios, correlacionando com os ganhos de resistência mecânica.

Tabela 10: Velocidade da onda ultrassônica nos traços estudados

\begin{tabular}{c|c|c|c|c|c|c|c|c|c}
\hline \multirow{2}{*}{$\begin{array}{c}\text { TEMPERATURA } \\
\text { DE CURA }\left({ }^{\circ} \mathbf{C}\right)\end{array}$} & \multicolumn{3}{|c|}{ RELOCIDADE DE PROPAGAÇÃO DA ONDA ULTRASSÔNICA (m/s) } \\
\cline { 2 - 10 } & $\begin{array}{c}\mathbf{1} \\
\mathbf{d i a}\end{array}$ & $\begin{array}{c}\mathbf{7} \\
\mathbf{d i a s}\end{array}$ & $\begin{array}{c}\mathbf{2 8} \\
\mathbf{d i a s}\end{array}$ & $\begin{array}{c}\mathbf{1} \\
\mathbf{d i a}\end{array}$ & $\begin{array}{c}\mathbf{7} \\
\mathbf{d i a s}\end{array}$ & $\begin{array}{c}\mathbf{2 8} \\
\mathbf{d i a s}\end{array}$ & $\begin{array}{c}\mathbf{1} \\
\mathbf{d i a}\end{array}$ & $\begin{array}{c}\mathbf{7} \\
\text { dias }\end{array}$ & $\begin{array}{c}\mathbf{2 8} \\
\text { dias }\end{array}$ \\
\hline 22,0 & 4761 & 4819 & 4866 & 4576 & 4597 & 4619 & 4310 & 4319 & 4444 \\
\hline 8,0 & 4040 & 4555 & 4728 & 4024 & 4395 & 4629 & 3891 & 4273 & 4494 \\
\hline 3,0 & 3649 & 4651 & 4773 & 3278 & 4405 & 4728 & 3344 & 4123 & 3944 \\
\hline 0,0 & 3603 & 4587 & 4683 & 3068 & 4566 & 4619 & 2497 & 4348 & 4494 \\
\hline$-3,0$ & 3952 & 4597 & 4819 & 2971 & 4415 & 4796 & 2230 & 4166 & 4619 \\
\hline
\end{tabular}

Os resultados apresentados na Tabela 10 evidenciam a evolução na velocidade de propagação da onda ultrassônica até a idade de 28 dias. Todavia, os ganhos foram diferentes em cada traço. Para a temperatura de 0 e $-3{ }^{\circ} \mathrm{C}$, o aumento dos valores entre o $1^{\circ}$ dia e o $28^{\circ}$ foi de $56 \%$, em média, sendo a mais alta encontrada no traço pobre, elevando sua velocidade de propagação de onda em até $100 \%$. Já o aumento da velocidade nos traços curados em $22{ }^{\circ} \mathrm{C}$ foi de $2 \%$, em média. Com isso, verificou-se que a cura em baixas temperaturas proporcionou maior taxa de evolução da velocidade da onda ultrassônica, atingindo, aos 28 dias, valores muito próximos aos curados em temperaturas mais elevadas. Liu et al. [33] verificaram o mesmo comportamento em matrizes curadas em condições extremas, tendo como consequência o aumento da solidez do material com a diminuição de temperatura.

No primeiro dia de idade, as misturas condicionadas em menores temperaturas apresentaram baixa velocidade de propagação da onda ultrassônica, pelo fato de obterem maior teor de umidade interna e apresentar vazios devido a solidificação das moléculas de água livre. Comparando os valores com os curados aos $22{ }^{\circ} \mathrm{C}$, observou-se uma queda de $26 \%$ (rico), $52 \%$ (intermediário) e $83 \%$ (pobre). A fim de facilitar a visualização dos resultados, elaborou-se uma curva correspondendo aos valores de velocidade da onda ultrassônica e o grau de maturidade das amostras (vide Figura 4).

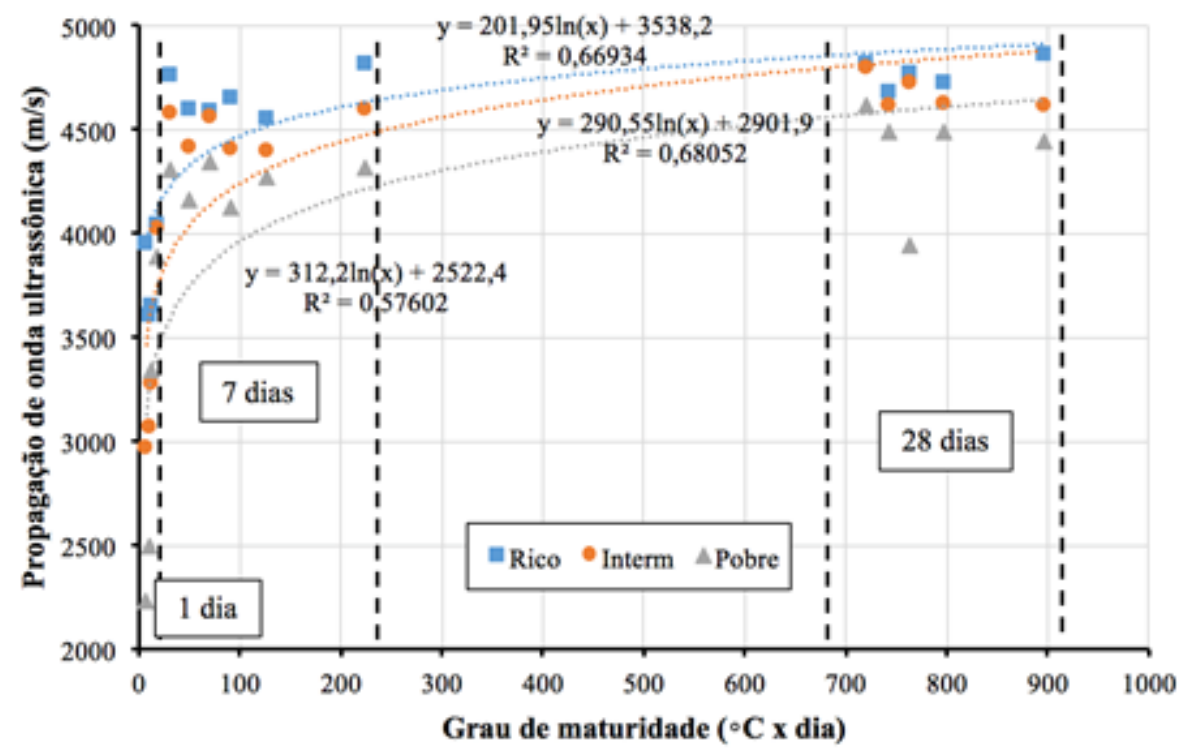

Figura 4: Propagação de onda ultrassônica em relação ao grau de maturidade

Por meio das curvas apresentadas na Figura 4, nota-se que os valores foram variados entre si, tendo uma correlação entre 0,58 a 0,68 . Também foi possível verificar que os resultados de propagação de onda ultrassônica apresentaram variação quando possuíam um GM inferior a $50{ }^{\circ} \mathrm{C} *$ dia, ou seja, nas primeiras idades, mostrando que os resultados, com baixo grau de maturidade, podem não ser previsíveis através deste 
modelo. Os resultados mais estáveis foram atingidos pelas misturas com maior grau de maturidade. Já nas idades de 7 e 28 dias, independente do GM, as amostras apresentaram valores semelhantes entre si.

Para os traços rico e intermediário, a velocidade de propagação da onda ultrassônica, em todas as temperaturas, foi superior a $4500 \mathrm{~m} / \mathrm{s}$, sendo classificados como excelentes frente aos valores determinados por Whitehurst [57]. Observa-se que as amostras curadas em $-3{ }^{\circ} \mathrm{C}$ obtiveram os maiores valores, ou seja, os traços com menor índice de vazios. Já para o traço pobre, todas as amostras foram classificadas como ótimas, pois seus valores permaneceram entre 3500 a $4500 \mathrm{~m} / \mathrm{s}$, com exceção dos exemplares curados em $-3{ }^{\circ} \mathrm{C}$. No traço pobre curado a $-3{ }^{\circ} \mathrm{C}$ a velocidade atingiu $4619 \mathrm{~m} / \mathrm{s}$, sendo classificada como excelente. Dessa forma, verifica-se que a cura de concretos em baixas temperaturas auxiliou nos ganhos da mistura, muitas vezes superando as amostras curadas em temperatura normativa ou estando equivalentes às mesmas.

\section{CONCLUSÕES}

Verificando os valores de investigação obtidos ao longo do estudo é notória a influência da temperatura de cura em concretos, independente da sua composição. Os efeitos foram consideráveis nas idades inicias da mistura, quando a mesma se encontrava em transição do estado fresco para o viscoelástico. $\mathrm{O}$ traço de concreto com maior consumo de cimento apresentou os melhores resultados nos primeiros 7 dias de análise, tanto nas condições extremas, quanto em temperatura ambiente. Nesse caso, conclui-se que a eficiência na hidratação do cimento em baixas temperaturas é diretamente proporcional ao consumo e relação água / cimento, pelo fato de haver uma maior distribuição do aglomerante pela matriz e diminuir o percurso em que a água-gel encontra para movimentar-se ao grão. A mistura com menor quantidade de cimento e exposta a menor temperatura obteve resistência nula, o que mostra a dificuldade de reações químicas no interior da matriz para o desenvolvimento do sólido.

A temperatura de cura influiu na difusão da água pela mistura, pois limitou seu escoamento. Mesmo assim, após o período de exposição às baixas temperaturas, as matrizes apresentaram ganhos de resistência mais expressivos. Esse comportamento foi ocasionado pela mudança do estado físico da água, passando de gel/sólido para líquido, melhorando a sua mobilidade no sistema e atingindo os grãos de cimento anidros. Essa hidratação residual teve como principal fator a diminuição da energia para mobilidade das moléculas da água.

Desse modo, a hidratação residual foi tão eficiente que as matrizes curadas inicialmente em temperaturas próximas a $0{ }^{\circ} \mathrm{C}$ obtiveram compacidade e resistências superiores as condicionas integralmente em $22{ }^{\circ} \mathrm{C}$. Nesse caso, o grau de maturidade não foi proporcional, onde aos 28 dias as matrizes com menor valor apresentaram resistências e velocidade de onda ultrassônica superior as com maior GM. Com isso, verificou-se o efeito positivo causado pelas baixas temperaturas nas idades inicias de cura de concretos com diferentes consumos de cimento, onde os reflexos foram mais evidentes em idades avançadas, sabendo-se que, nos 7 dias inicias, houve dificuldade no desenvolvimento das propriedades mecânicas, necessitando de maiores cuidados.

\section{AGRADECIMENTOS}

Os autores deste artigo agradecem ao Itt Performance (Instituto Tecnológico em Desempenho e Construção Civil) da Unisinos o apoio para elaborar a pesquisa.

\section{BIBLIOGRAFIA}

[1] CARMONA FILHO, A., CARMONA, T., "Fissuração nas estruturas de concreto", Boletim Técnico ALCONPAT Internacional, 2013.

[2] SANTAMARÍA, A., ORBE, A., SAN JOSÉ, J. T., GONZÁLEZ, J. J., "A study on the durability of structural concrete incorporating electric steelmaking slags", Construction and Building Materials, v. 161, pp. 94-111, 2018.

[3] VLAHOVIC, M. M., MARTINOVIC, S. P., BOLJANAC, T. J., et al., "Durability of sulfur concrete in various aggressive environments", Construction and Building Materials v. 25, pp. 3926-3934, 2011.

[4] CALLISTER Jr., W. D., RETHWISCH, D. G., "Ciência e Engenharia de Materiais. Uma Introdução", São Paulo, GEN LTC ATLAS Editora, 9 $9^{\mathrm{a}}$ Edição, pp. 866, 2016.

[5] RICHARDSON, M., "Degradation of concrete in cold weather conditions", Durability of concrete and cement composites, The Institute of Materials, Minerals \& Mining, New York, Cap. 8, 2007.

[6] GJORV, O. E., "Projeto da durabilidade de estruturas de concreto em ambientes de severa agressividade", São Paulo, Oficina de Textos (Supervisão Paulo Helene e Enio Pazini Figueiredo), pp. 240, 2015. 
[7] PAGE, C. L., PAGE, M. M., "Durability of concrete and cement composites", The Institute of Materials, Minerals \& Mining, New York, 2007.

[8] METHA, P. K., MONTHEIRO, P. J. M., “Concreto, microestrutura e propriedades e materiais”, São Paulo. IBRACON, pp. 674, 2014.

[9] REBMANN, M. S., "Robustez de concretos com baixo consumo de cimento Portland: desvios no proporcionamento e variabilidade granulométrica e morfológica dos agregados”, Tese de Doutorado. Escola Politécnica da Universidade de São Paulo. Departamento de Engenharia e Construção, São Paulo, 2017.

[10] EHRENBRING, H. Z., "Comportamento de concretos reforçados com microfibras de polipropileno (PP), álcool polivinílico (PVA) e recicladas de poliéster (POL) em relação à retração por secagem restringida e às propriedades mecânicas", Dissertação de Mestrado, Programa de Pós-Graduação em Engenharia Civil da Universidade do Vale do Rio dos Sinos, São Leopoldo, 2017.

[11] NEVILLE, A. M., "Propriedades do concreto", Tradução Eng. Salvador E. Giammusso. São Paulo: Pini, pp. 828, 1997.

[12] GARCIA, M. L., SÁNCHEZ, M. I., "Pozzolanic reaction of a spent fluid catalytic cracking catalyst in FCC-cement mortars”, Journal of Thermal Analysis and Calorimetry, V. 90, pp. 443-447, 2007.

[13] HWANG, H. J., PARK, H. G., HONG, G. H., et al., "Immediate and Long-Term Deflections of Reinforced Concrete Slabs Affected by Early-Age Loading and Low Temperature", ACI Structural Journal v. 109 no. 3, pp. 413-422, 2012.

[14] LIMA, S. M., LIBÓRIO, J. B. L., "Concreto de alto desempenho em ambientes com baixas temperaturas”, Cadernos de Engenharia de Estruturas, São Carlos, v. 10, n. 43, p. 55-73, 2008.

[15] SORIANO, L., MONZÓ, J., BONILLA, M., et al., "Effect of pozzolans on the hydration process of Portland cement cured at low temperatures", Cement \& Concrete Composites, No. 42, pp. 41-48, 2013.

[16] CASTRO, A. L., "A influência do tipo de cimento no desempenho de concretos avançados formulados a partir do método de dosagem computacional”, Cerâmica, v. 57, n. 341, 2011.

[17] KRAAI, P. P., “A proposed test to determine the cracking potential due to drying shrinkage of concrete", Concrete Construction, v. 30, n. 9, pp. 775-778, 1985.

[18] KIM, J. K., MOON, Y. H., "Compressive strength development of concrete with different curing time and temperature", Cement \& Concrete Research, v. 28, pp. 1761-73, 1998.

[19] HUSEM, M., GOZUTOK, S., "The effects of low temperature on the compressive strength of ordinary and high performance concrete", Construction and Building Materials, v. 19, pp. 49-53, 2005.

[20] LOTHENBACH, B., MATSCHEI, T., MÖSCHNER, G., et al., "Thermodynamic modelling of the effect of temperature on the hydration and porosity of Portland cement", Cement \& Concrete Research, V. 38, pp. 1-18, 2008.

[21] ANGULISKI DA LUZ, C., HOOTON, R. D., "Influence of curing temperature on the process of hydration of supersulfated cements at early age", Cement and Concrete Research, v. 77, pp. 69-75, 2015.

[22] PAYA, J., MONZÓ, J., BORRACHERO, M. V., "Fluid catalytic cracking catalyst residue (FC3R). An excellent mineral by-product for improving early strength development of cement mixtures", Cement \& Concrete Research, V. 29, pp. 1773-1779, 1999.

[23] POWERS, T. C., HELMUTH, R. A., "Theory of volume changes in hardened Portlandcement past during freezing", Proceedings Highway Research Board, v. 32, p. 285-297, 1953.

[24] MANIKANDAN, R., RAMAMURTHY, K., "Effect of curing method on characteristics of cold bonded fly ash aggregates", Cement and Concrete Composites, n 9, pp. 848-853, 2008.

[25] FUNCHAL, F. E., "Modelo de previsão da elevação adiabática de temperatura do concreto através de redes neurais artificiais", 2004

[26] CECCONELO, V., TUTIKIAN, B. "A influência das baixas temperaturas na evolução da resistência do concreto”, São Paulo. IBRACON, V. 5, No. 1, pp. 68-83, 2012.

[27] CHAKRABORTY, A. K., DUTTA, S. C., "Study on silica fume modified mortar with various Indian cements cured at different temperatures", Building Environment, v. 36, pp. 375-82, 2001.

[28] ESCALANTE-GARCÍA, J. I., SHARP, J. H., "The microstructure and mechanical properties of blended cements hydrated at various temperatures”, Cement \& Concrete Research, v. 31, pp. 695-702, 2001.

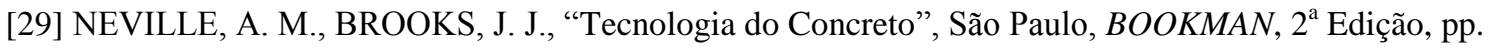
472, 2013.

[30] PIGNEON, M., PLEAU, R., "Durability of Concrete in Cold Climates", E \& FN Spon, 1998. 
[31] BURG, R. G., "The Influence of Casting and Curing Temperature on the Properties of Fresh and Hardened Concrete", Portlan Cement Association, ISBN 0-89312-143-6, 1996.

[32] POWERS, T. C., "The air requirement of frost-resistance concrete", Proceedings Highway Research Board, v. 29, p. 184-211, 1949.

[33] LIU, X., ZHANG, M. H., CHIA, K. S., et al., "Mechanical properties of ultra-lightweight cement composite at low temperatures of 0 to $-60^{\circ} \mathrm{C}^{\prime}$, Cement and Concrete Composites, v. 16, pp. 1-22, 2016.

[34] XU, L., WU, K., R€OßLER, C., et al., "Influence of curing temperatures on the hydration of calcium aluminate cement/Portland cement/calcium sulfate blends", Cement and Concrete Composites v. 80 pp. 298306, 2017.

[35] TUTIKIAN, B. F., CECCONELLO, V., "The influence of low temperature on the evolution of concrete strength”, Revista IBRACON de Estruturas e Materiais, v. 5, pp. 68-83, 2012.

[36] BOLINA, F., CHRIST, R., METZLER, A., et al., "Comparison of the Fire Resistance of two Structural Wall Systems in Light Steel Framing", DYNA (MEDELLÍN), v. 84, 2017.

[37] ACI 306R-88. Cold weather concreting. MCP 2003. ACI Manual of Concrete Practice; 2003.

[38] EHE-08. Instrucción de Hormigón Estructural. Ministerio de Fomento; 2008.

[39] ABNT (2004), NBR 14931: Execução de estruturas de concreto - Procedimento. Rio de Janeiro.

[40] NMCAI, C. K., "Cold Weather Concreting Admixtures", Cement and Concrete Composites, V. 20, pp. 21- 128, 1998.

[41] LAGUNA, L. A., IKEMATSU, P., "Influência térmica na desforma do concreto", Téchne. 2015.

[42] VANCURAA, M., MACDONALD, K., KHAZANOVICHA, L., "Microscopic analysis of paste and aggregate distresses in pervious concrete in a wet, hard freeze climate", Cement and Concrete Composites, $\mathrm{n}$ 10, pp. 1080-1085, 2011.

[43] HAWKES, J., "The Effects of Pouring Concrete in Cold Weather", Power Blanket, 2017.

[44] ABNT (2015), NBR 5738: Moldagem e cura de corpos-de-prova cilíndricos ou prismáticos de concreto. Rio de Janeiro.

[45] ABNT (1998), NBR.NM 23: Cimento - Determinação de massa especifica. Rio de Janeiro.

[46] ABNT (2006), NBR.NM 45: Determinação de massa unitária de agregados. Rio de Janeiro.

[47] ABNT (2009), NBR NM 52: Agregado miúdo - Determinação da massa especifica e massa especifica aparente. Rio de Janeiro.

[48] ABNT (2009), NBR 7211. Agregados para concretos - Especificação. Rio de Janeiro.

[49] ABNT (2002), NBR.NM 53: Agregado graúdo - Determinação de massa especifica aparente e absorção de água. Rio de Janeiro.

[50] TUTIKIAN, B. F., HELENE, P. "Dosagem dos Concretos de Cimento Portland", Concreto: Ciência e Tecnologia, v. 1, n. 1927, pp. 439-471, 2011.

[51] ABNT (2012), NBR 7212: Execução de concreto dosado em Central- Procedimento. Rio de Janeiro.

[52] ABNT (2007), NBR 5739- Concreto - Ensaio de compressão de corpos-de-prova cilíndricos. Rio de Janeiro.

[53] ACI 325.12R-02. Guide for Design of Jointed Concrete Pavements for Streets and Local Roads. MCP 2002. ACI Manual of Concrete Practice; 2002.

[54] ABNT (2015), NBR 7222. Determinação da resistência a tração por compressão diametral de corpos de prova cilíndricos. Rio de Janeiro.

[55] IS 13311: Non-destructive testing of concrete - Methods of Test - Part 1: Ultrasonic pulse velocity. New Delhi: Bureau Of Indian Standards, 1992.

[56] ABNT (2013), NBR. NM 8802: Determinação da velocidade de onda ultrassônica. Rio de Janeiro.

[57] WHITENHURST, E. A., "Evoluation of concrete properties from sonic tests", ACI Monografia, American Concrete Institute/Iowa State University Press, Detroit, n. 2, pp. 94, 1966.

[58] YAN, J. B., XIE, J., "Behaviours of reinforced concrete beams under low temperatures", Construction and Building Materials, v. 141, pp. 410-425, 2017. 


\section{ORCID}

Alessandra Wirth

https://orcid.org/0000-0002-1950-0342

Bernardo Fonseca Tutikian

https://orcid.org/0000-0003-1319-0547

Daniel Reis Medeiros

https://orcid.org/0000-0002-6097-5008

Hinoel Zamis Ehrenbring

https://orcid.org/0000-0002-0339-9825

Rafael Muller Munchen

https://orcid.org/0000-0003-0173-2353 